\title{
A Quasi-Conservative Discontinuous Finite Element Method for Nuclear Reactor Point Kinetics Model ${ }^{1}$
}

C.R. GARCÍA, D.S. DOMÍNGUEZ, R.C. BARROS, Departamento de Modelagem Computacional, Instituto Politécnico - IPRJ, Universidade do Estado do Rio de Janeiro, Cx.P. 97282, 28601-970 Nova Friburgo, RJ, Brazil.

\begin{abstract}
The point reactor kinetics model is an initial value problem characterized by a system of ordinary differential equations that describe the time dependence of the neutron population within a nuclear reactor core and the decay of the delayed neutron precursors. This approach is useful as it permits to develop efficient algorithms for real time simulation training in nuclear power plants. In this paper, we describe a quasi-conservative linear discontinuous finite element method to numerically solve the nuclear reactor point kinetics equations with six groups of delayed neutron precursors. This method is based on the expansion of the solution in Legendre polynomials within each time step. As we truncate the expansion in the first degree Legendre polynomial, we refer to the offered method as the linear discontinuous (LD) method. In deriving the LD discretized equations, we find the discretized balance equations for the $\ell$ order moments, $\ell=0,1$. For the case of prescribed reactivity change as a linear function of time, in the first-order moment equation, we are left with the second-order moment that we need to approximate. Therefore, we obtain a quasi-conservative numerical scheme, as the discretized balance equation for the first-order moment is approximated. Moreover, considering linear expansion of the solution within each time step, we obtain auxiliary equations that, together with the balance equations, form a system of fourteen algebraic linear equations in fourteen unknowns for each time step. We show numerical results to two typical model problems to illustrate the LD method's accuracy.
\end{abstract}

\section{Introduction}

Accurate simulation of the time-dependent behavior of neutron population in a fission chain-reacting system in response to either a planed change in the system conditions or to an abnormal condition is essential for the safe operation of nuclear reactors. The dynamics of a nuclear reactor under normal operation is governed primarily by the characteristics of the delayed emission of neutrons from the decay of fission products, which can be modeled by defining six effective groups of delayed neutron precursor fission products. The conventional point kinetics model for nuclear reactor physics is an initial value problem, composed by seven ordinary differential equations, that are obtained by assuming separation of variables solutions

${ }^{1}$ The authors are grateful to CNPq for the financial support. 
of the time-dependent neutron diffusion equation coupled with six-group delayed neutron precursor equations. The limiting assumption for the accurate simulation of the point kinetics equations is the assumption of a constant spatial shape, which is expected to be reasonable for transients caused by uniform changes in reactor proprieties.

In practice, numerical methods are used to solve the neutron dynamics equations, and a class of accurate and efficient algorithms has been developed for over twenty years $[2,3,4]$. In this paper, we describe a discontinuous finite element method to numerically solve the nuclear reactor point kinetics equations with six groups of delayed neutron precursors. This method, extended from the applications to the neutron transport equation in the discrete ordinates formulation, is based on the expansion of the solution in Legendre polynomials within each time step. As we truncate the expansion in the first degree Legendre polynomial, we refer to the offered method as the linear discontinuous (LD) method. In deriving the LD discretized equations, we first find the discretized balance equations for the $\ell^{\text {th }}$ order moments, $\ell=0,1$. For the case of prescribed reactivity change as a linear function of time, in the first order moment equation, we are left with the second order moment that we need to approximate, and thus, obtain a quasi-conservative numerical scheme, as the discretized balance equation for the first order moment $\ell=1$ is approximated.

Moreover, considering the linear expansion of the solution within each time step, we obtain auxiliary equations that, together with the balance equations, form a system of fourteen algebraic linear equations in fourteen unknowns for each time step. We consider five different schemes to approximate the second-order moment of neutron density and we describe them in section 2 . In section 3 we show the numerical results to two test problems. We consider one test problem with the purpose of testing the accuracy of the different schemes to approximate the second-order moment. In the test problem No. 2 we compare the present quasi-conservative LD finite element method for nuclear reactor point kinetics model with recent numerical methods published in the literature. In section 4 we list a number of general conclusions and suggestions for future works.

\section{Quasi-Conservative Linear Discontinuous Finite Element Method}

The point kinetics equations with six-groups of delayed neutron precursors [5] can be written as

$$
\begin{aligned}
\frac{d N(t)}{d t} & =\left[\frac{\rho(t)-\beta}{\Lambda}\right] N(t)+\sum_{m=1}^{6} \lambda_{m} C_{m}(t), \\
\frac{d C_{m}(t)}{d t} & =\frac{\beta_{m} N(t)}{\Lambda}-\lambda_{m} C_{m}(t), m=1: 6,
\end{aligned}
$$

where 
$N$ - neutron density,

$C_{m}$ - concentration of delayed neutron precursors of the $m^{\text {th }}$ group,

$\beta_{m}$ - fraction of delayed neutrons emitted by the $m^{\text {th }}$ group,

$\beta$ - total fraction of fission neutrons, that are delayed,

$\Lambda$ - mean generation time between neutron birth and subsequent absorption inducing fission,

$\lambda_{m}$ - decay constant of the $m^{\text {th }}$ group,

$\rho$ - reactivity.

We assume that the reactivity $\rho(t)$ is a linear function of time [4], that is

$$
\rho(t)=\rho_{0}+2 \nu \beta \frac{\left(t-t_{i}\right)}{s},
$$

where

$s$ - width of the time step in a uniform grid,

$\nu$ - reactivity insertion control as a function of time,

$\rho_{0}$ - initial reactivity.

For initial conditions $t=0$ we consider normalized value of neutron density $N(0)=1$. Therefore in accordance with equation (2.2), we obtain

$$
C_{m}(0)=\frac{\beta_{m}}{\lambda_{m} \Lambda}, m=1: 6 .
$$

Considering constant physical properties within each time step, we divide the time domain $\mathrm{T}$ into elements $\mathrm{S}_{i}, i=1: \mathrm{I}$. To find the discretized balanced equations for the $\ell^{\text {th }}$ order moments within each element $\mathrm{S}$, we apply the operator

$$
\frac{(2 l+1)}{s} \int_{t_{i-1 / 2}}^{t_{i+1 / 2}} P_{\ell}\left[\frac{2\left(t-t_{i}\right)}{s}\right] d t
$$

to equations (2.1) and (2.2). Here, we have defined

$t_{i}=\left(t_{i-1 / 2}+t_{i+1 / 2}\right) / 2$,

$P_{\ell}$ - the $\ell^{\text {th }}$ degree Legendre polynomial,

$s$ - uniform width of element $\mathrm{S}_{i}$.

By setting $\ell=0$ in equation (2.5) and applying the resulting operator to equation (2.1), we obtain the zero-th order discretized balance equation

$$
\frac{N_{i+1 / 2}-N_{i-1 / 2}}{s}=\frac{\mathcal{L}_{i}}{\Lambda}-\frac{\beta}{\Lambda} \bar{N}_{i}+\sum_{m=1}^{6} \lambda_{m} \bar{C}_{m, i}
$$

where we have defined the average quantities

$$
\bar{N}_{i}=\frac{1}{s} \int_{t_{i-1 / 2}}^{t_{i+1 / 2}} N(t) d t
$$


and

$$
\bar{C}_{m, i}=\frac{1}{s} \int_{t_{i-1 / 2}}^{t_{i+1 / 2}} C_{m}(t) d t
$$

and the integral quantity

$$
\mathcal{L}_{i}=\frac{1}{s} \int_{t_{i-1 / 2}}^{t_{i+1 / 2}}\left[\rho_{0}+2 \nu \beta \frac{\left(t-t_{i}\right)}{s}\right] N(t) d t,
$$

that is equivalent to

$$
\mathcal{L}_{i}=\rho_{0} \bar{N}_{i}+\frac{\nu \beta}{3} \hat{N}_{i},
$$

where we have defined the first order moment of the neutron density

$$
\hat{N}_{i}=\frac{3}{s} \int_{t_{i-1 / 2}}^{t_{i+1 / 2}} \frac{2\left(t-t_{i}\right)}{s} N(t) d t=\frac{6}{s^{2}} \int_{t_{i-1 / 2}}^{t_{i+1 / 2}}\left(t-t_{i}\right) N(t) d t .
$$

By substituting expression (2.7) into equation (2.6), we obtain

$$
\frac{N_{i+1 / 2}-N_{i-1 / 2}}{s}=\frac{\left(\rho_{0}-\beta\right)}{\Lambda} \bar{N}_{i}+\frac{\nu \beta}{3 \Lambda} \hat{N}_{i}+\sum_{m=1}^{6} \lambda_{m} \bar{C}_{m, i}
$$

Now we set $\ell=0$ in equation (2.5) and apply the resulting operator to equation (2.2). The result is

$$
\frac{C_{m, i+1 / 2}-C_{m, i-1 / 2}}{s}=-\lambda_{m} \bar{C}_{m, i}+\frac{\beta_{m}}{\Lambda} \bar{N}_{i}, m=1: 6 .
$$

Furthermore, to obtain the first-order discretized balance equations, we apply operator (2.5) with $\ell=1$ to equations (2.1) and (2.2). By applying operator (2.5) with $\ell=1$ to equation (2.1) we obtain

$$
\frac{3\left[N_{i+1 / 2}+N_{i-1 / 2}-2 \bar{N}_{i}\right]}{s}=\frac{\left(\rho_{0}-\beta\right)}{\Lambda} \hat{N}_{i}+\frac{\nu \beta}{\Lambda} \bar{N}_{i}+\frac{2 \nu \beta}{5 \Lambda} \tilde{N}_{i}+\sum_{m=1}^{6} \lambda_{m} \bar{C}_{m, i}
$$

where we have defined the second order moment of the neutron density

$$
\tilde{N}_{i}=\frac{5}{s} \int_{t_{i-1 / 2}}^{t_{i+1 / 2}}\left\{\frac{3}{2}\left[\frac{2\left(t-t_{i}\right)}{s}\right]^{2}-\frac{1}{2}\right\} N(t) d t
$$

or equivalently

$$
\tilde{N}_{i}=\frac{30}{s^{3}} \int_{t_{i-1 / 2}}^{t_{i+1 / 2}}\left(t-t_{i}\right)^{2} N(t) d t-\frac{5}{2} \bar{N}_{i} .
$$

Operator (2.5) applied to equation (2.2) with $\ell=1$ implies

$$
\frac{3\left[C_{m, i+1 / 2}+C_{m, i-1 / 2}-2 \bar{C}_{m, i}\right]}{s}=\frac{\beta_{m}}{\Lambda} \hat{N}_{i}-\lambda_{m} \hat{C}_{m, i}, m=1: 6,
$$


where we have defined

$$
\hat{C}_{m, i}=\frac{3}{s} \int_{t_{i-1 / 2}}^{t_{i+1 / 2}} \frac{2\left(t-t_{i}\right)}{s} C_{m}(t) d t=\frac{6}{s^{2}} \int_{t_{i-1 / 2}}^{t_{i+1 / 2}}\left(t-t_{i}\right) C_{m}(t) d t .
$$

For $m=1: 6$, equations (2.8), (2.9), (2.10) and (2.12) form a system of fourteen algebraic linear equations in twenty-two unknowns, namely $N_{i+1 / 2}, \bar{N}_{i}, \hat{N}_{i}, \tilde{N}_{i}$, $C_{m, i+1 / 2}, \bar{C}_{m, i}$ and $\hat{C}_{m, i}, m=1: 6$. Moreover, we use the linear expansions

$$
N(t) \cong \bar{N}_{i}+\frac{2}{s}\left(t-t_{i}\right) \hat{N}_{i}
$$

and

$$
C_{m}(t) \cong \bar{C}_{m, i}+\frac{2}{s}\left(t-t_{i}\right) \hat{C}_{m, i}, m=1: 6,
$$

where we consider $t=t_{i+1 / 2}$ and hence obtain the auxiliary equations

$$
N_{i+1 / 2}=\bar{N}_{i}+\hat{N}_{i}
$$

and

$$
C_{m, i+1 / 2}=\bar{C}_{m, i+1 / 2}+\hat{C}_{m, i+1 / 2}, m=1: 6 .
$$

By substituting the auxiliary equations (2.15) and (2.16) into the discretized balance equations $(2.8),(2.9),(2.10)$ and $(2.12)$, we obtain

$$
\begin{gathered}
{\left[1-\frac{\left(\rho_{0}-\beta\right) s}{\Lambda}\right] \bar{N}_{i}+\left[1-\frac{\nu \beta s}{3 \Lambda}\right] \hat{N}_{i}-s \sum_{m=1}^{6} \lambda_{m} \bar{C}_{m, i}=N_{i-1 / 2}} \\
-\left[\frac{\beta_{m} s}{\Lambda}\right] \bar{N}_{i}+\left[1+\lambda_{m} s\right] \bar{C}_{m, i}+\hat{C}_{m, i}=C_{m, i-1 / 2}, m=1: 6, \\
{\left[3+\frac{s \nu \beta}{\Lambda}\right] \bar{N}_{i}-\left[3-\frac{s\left(\rho_{0}-\beta\right)}{\Lambda}\right] \hat{N}_{i}+s \sum_{m=1}^{6} \lambda_{m} \hat{C}_{m, i}+\frac{2 \nu \beta s}{5 \Lambda} \tilde{N}_{i}=3 N_{i-1 / 2}} \\
-\left[\frac{s \beta_{m}}{\Lambda}\right] \hat{N}_{i}-3 \bar{C}_{m, i}+\left[3+\lambda_{m} s\right] \hat{C}_{m, i}=-3 C_{m, i-1 / 2}, m=1: 6 .
\end{gathered}
$$

For each time step, equations (2.17-2.20) form a system of fourteen algebraic linear equations in fifteen unknowns, namely $\bar{N}_{i}, \hat{N}_{i}, \tilde{N}_{i}, \bar{C}_{m, i}$ and $\hat{C}_{m, i}$. We are still left with the second-order moment $\tilde{N}_{i}$ in equation $(2.19)$, which we need to approximate, and thus obtain a quasi-conservative numerical scheme. In this paper, we consider five different schemes that we list in Table 1.

We modify the coefficients in equation (2.19) by considering each one of the approximations listed in Table 1 and we advance in time by solving the system of equations (2.17-2.20) and then using the auxiliary equations (2.15) and (2.16). To solve system (2.17-2.20) we use Gaussian elimination with scaled pivoting and backward substitution [1]. 
Table 1: Numerical approximations for the second order moment $\tilde{N}_{i}$.

\begin{tabular}{|c|l|}
\hline Numerical Schemes & Numerical approximations for $\tilde{N}_{i}$ \\
\hline 1 & $\begin{array}{l}\text { We simply neglect the second order moment } \tilde{N}_{i} \text { in the bal- } \\
\text { ance equation }(2.19) .\end{array}$ \\
\hline 2 & $\begin{array}{l}\text { We approximate } \tilde{N}_{i} \text { by }-\frac{5}{2} \bar{N}_{i}, \text { i.e., we neglect the integral } \\
\text { term in equation }(2.11) .\end{array}$ \\
\hline 3 & We approximate $\tilde{N}_{i}$ by the average, $\tilde{N}_{i}=\frac{\left(N_{i+1 / 2}+N_{i-1 / 2}\right)}{2}$. \\
\hline 4 & $\begin{array}{l}\text { We consider the numerical explicit approximation, } \\
\tilde{N}_{i}=N_{i-1 / 2} .\end{array}$ \\
\hline 5 & We approximate $\tilde{N}_{i}$ by the first-order moment, $\tilde{N}_{i}=\hat{N}_{i}$. \\
\hline
\end{tabular}

\section{Numerical Results}

At this point we consider two model problems with the purpose of testing the accuracy of the approximations in the present LD method for numerically solving the system of seven coupled ordinary differential equations of the point reactor kinetics model given in equations(2.1-2.2).

\subsection{Test problem No. 1}

In this test problem the reactor power level is supposed to increase very rapidly. The reactor nuclear data are listed in Table 2. The reactivity insertion is given by equation (2.3) and $0 \leq \mathrm{T} \leq 10$ seconds. The rate of reactivity insertion is 0.02 dollar/second (\$/sec.) and the initial value of inserted reactivity $\rho_{0}$ is $0.4615 \$$. Moreover the initial conditions are $N(0)=1$ and equation (2.4). Table 3 displays the fourth order Runge Kutta [1] reference results, generated on a fine time grid (time step $=0.1$ second).

We ran model problem No. 1 with time steps of 1 and 2 seconds for the five numerical schemes of quasi-conservative LD finite element method, viz Table 1. In Tables 4 and 5 we show the relative deviations of neutron density and precursor concentrations with respect to the reference solution (viz Table 3 ) for the five schemes.

As we see in Tables 4 and 5 , for wide time steps, we obtain very good agreement with respect to the fine grid reference results listed in Table 3. That is, the relative deviations are all less than $1 \%$, except for numerical scheme number 2 (Table 1 ) that generated the least accurate results. 
Table 2: Nuclear data for test problems No. 1.

\begin{tabular}{|c|l|l|}
\hline Group $m$ & $\lambda_{m}$ & $\beta_{m}$ \\
\hline \hline 1 & 0.0124 & 0.000215 \\
\hline 2 & 0.0305 & 0.001424 \\
\hline 3 & 0.1110 & 0.001274 \\
\hline 4 & 0.3010 & 0.002568 \\
\hline 5 & 1.1400 & 0.0007488 \\
\hline 6 & 3.0100 & 0.000273 \\
\hline \hline$\Lambda$ & \multicolumn{3}{|l|}{$\beta=\sum_{m=1}^{6}$} & $\beta_{m}$ \\
\hline 0.001 & 0.0065 \\
\hline
\end{tabular}

Table 3: Neutron density and precursor concentrations at 10 seconds, for test problem No. 1 (Time step equal to 0.1 second in the fourth order Runge Kutta method).

\begin{tabular}{|c|c|c|c|c|c|c|c|}
\hline Density & $N$ & $C_{1}$ & $C_{2}$ & $C_{3}$ & $C_{4}$ & $C_{5}$ & $C_{6}$ \\
\hline Ref. Results & 26.328 & 32.044 & 139.782 & 80.815 & 107.711 & 13.253 & 2.135 \\
\hline
\end{tabular}

Table 4: Relative deviations (\%) with respect to the reference results listed in Table 3 (Time step $s=1$ second).

\begin{tabular}{|c|c|c|c|c|c|c|c|}
\hline Schemes & $\Delta N / N$ & $\Delta_{1}^{*}$ & $\Delta_{2}^{*}$ & $\Delta_{3}^{*}$ & $\Delta_{4}^{*}$ & $\Delta_{5}^{*}$ & $\Delta_{6}^{*}$ \\
\hline 1 & 0.08 & -0.02 & 0.03 & 0.03 & 0.02 & 0.01 & 0.02 \\
\hline 2 & 0.26 & -0.21 & -0.30 & -0.40 & -0.44 & -0.41 & -0.29 \\
\hline 3 & 0.01 & 0.11 & 0.16 & 0.20 & 0.21 & 0.18 & 0.14 \\
\hline 4 & 0.02 & 0.10 & 0.14 & 0.18 & 0.18 & 0.16 & 0.12 \\
\hline 5 & 0.06 & 0.04 & 0.05 & 0.06 & 0.06 & 0.04 & 0.04 \\
\hline
\end{tabular}

${ }^{(*)} \Delta_{i}=\Delta C_{i} / C_{i}$

Table 5: Relative deviations (\%) with respect to the reference results listed in Table 3 (Time step $s=2$ second).

\begin{tabular}{|c|c|c|c|c|c|c|c|}
\hline Schemes & $\Delta N / N$ & $\Delta_{1}^{*}$ & $\Delta_{2}^{*}$ & $\Delta_{3}^{*}$ & $\Delta_{4}^{*}$ & $\Delta_{5}^{*}$ & $\Delta_{6}^{*}$ \\
\hline 1 & 0.54 & 0.19 & 0.26 & 0.28 & 0.23 & 0.19 & 0.27 \\
\hline 2 & 0.61 & -0.73 & -1.06 & -1.39 & -1.50 & -1.23 & -0.65 \\
\hline 3 & 0.51 & 0.56 & 0.80 & 0.96 & 0.93 & 0.77 & 0.64 \\
\hline 4 & 0.54 & 0.44 & 0.63 & 0.76 & 0.72 & 0.59 & 0.53 \\
\hline 5 & 0.51 & 0.31 & 0.44 & 0.51 & 0.47 & 0.38 & 0.39 \\
\hline
\end{tabular}

${ }^{(*)} \Delta_{i}=\Delta C_{i} / C_{i}$ 


\section{2. $\quad$ Test problem No. 2}

At this point we compare the present quasi-conservative LD finite element method with numerical methods offered in references [3, 4] for nuclear reactor point kinetics model. Here the nuclear data are listed in Table 6 , and we consider zig-zag shape reactivity insertion, that is, in $\mathrm{T}<5$ sec. and $\mathrm{T}>15$ sec. the rate of reactivity insertion is $0.1 \$ /$ sec., and in 5 sec. $<\mathrm{T}<15$ sec. the rate of reactivity insertion is $-0.1 \$$ sec., and the initial value of inserted reactivity $\rho_{0}$ is $0 \$$. Moreover, the remaining initial conditions are $N(0)=1$ and equation (2.4).

Table 6: Nuclear data for test problem No. 2.

\begin{tabular}{|c|l|l|}
\hline Group $m$ & $\lambda_{m}$ & $\beta_{m}$ \\
\hline \hline 1 & 0.0127 & 0.000266 \\
\hline 2 & 0.0317 & 0.001491 \\
\hline 3 & 0.1150 & 0.001316 \\
\hline 4 & 0.3110 & 0.002849 \\
\hline 5 & 1.4000 & 0.000896 \\
\hline 6 & 3.8700 & 0.000182 \\
\hline \hline$\Lambda$ & $\beta=\sum_{m=1}^{6} \beta_{m}$ \\
\hline 0.00002 & 0.007 \\
\hline
\end{tabular}

In Table 7 we show the neutron density results and relative deviations with respect to the reference solution given in Ref. [4] for different methods. From this table we conclude that the relative deviations calculated with the offered method with the same time step with respect to the reference solution are much smaller in comparison with the other methods considered. Moreover, the present QuasiConservative Linear Discontinuous Finite Element method models the same test problem using a larger time step with similar accuracy. This characteristic indicates that the offered method has good efficiency in wide time step calculations. 
Table 7: Neutron density results and relative deviations (\%), for the three methods.

\begin{tabular}{|l|l|l|l|l|l|l|l|l|l|}
\hline $\begin{array}{l}\text { Time } \\
(\mathrm{sec})\end{array}$ & $\begin{array}{l}N^{\mathrm{a}} \\
\text { ref. } \\
\text { sol. }\end{array}$ & $\begin{array}{l}N^{\mathrm{b}} \\
\text { Ref. } \\
{[4]}\end{array}$ & $\delta(\%)^{\mathrm{c}}$ & $\begin{array}{l}N^{\mathrm{d}} \\
\text { Ref. } \\
{[3]}\end{array}$ & $\delta(\%)^{\mathrm{e}}$ & $\begin{array}{l}N^{\mathrm{f}} \mathrm{LD} \\
s=0.1\end{array}$ & $\begin{array}{l}\delta(\%)^{\mathrm{g}} \\
\mathrm{LD} \\
s=0.1\end{array}$ & $\begin{array}{l}N^{\mathrm{h}} \\
\mathrm{LD} \\
s=1\end{array}$ & $\begin{array}{l}\delta(\%)^{\mathrm{i}} \\
\text { LD } \\
s=1\end{array}$ \\
\hline 2 & 1.338 & 1.339 & -0.067 & 1.343 & -0.379 & 1.338 & 0.003 & 1.334 & 0.336 \\
\hline 4 & 2.228 & 2.231 & -0.132 & 2.248 & -0.905 & 2.228 & 0.003 & 2.213 & 0.702 \\
\hline 6 & 3.239 & 3.232 & 0.190 & 3.254 & -0.482 & 3.239 & -0.007 & 3.239 & -0.014 \\
\hline 8 & 2.656 & 2.654 & 0.085 & 2.656 & 0.024 & 2.656 & -0.002 & 2.650 & 0.218 \\
\hline 10 & 2.021 & 2.021 & -0.006 & 2.016 & 0.233 & 2.021 & -0.002 & 2.016 & 0.220 \\
\hline 12 & 1.502 & 1.503 & -0.047 & 1.497 & 0.337 & 1.502 & -0.002 & 1.499 & 0.190 \\
\hline 14 & 1.112 & 1.113 & -0.069 & 1.108 & 0.387 & 1.112 & -0.001 & 1.111 & 0.157 \\
\hline 16 & 0.962 & 0.962 & -0.106 & 0.959 & 0.250 & 0.962 & -0.002 & 0.962 & -0.014 \\
\hline 18 & 1.042 & 1.043 & -0.132 & 1.041 & 0.070 & 1.042 & -0.003 & 1.041 & 0.040 \\
\hline 20 & 1.247 & 1.249 & -0.164 & 1.248 & -0.129 & 1.247 & -0.002 & 1.245 & 0.137 \\
\hline
\end{tabular}

(a) neutron density results given as reference solution in Ref. [4].

(b) neutron density results generated by the method described in Ref. [4], with $s=0.1$ second.

(c) relative deviations of the neutron density results generated by the method described in Ref. [4], with respect to the reference results.

(d) neutron density results generated by the method described in Ref. [3], with $s=0.1$ second.

(e) relative deviations of the neutron density results generated by the method described in Ref.[3], with respect to the reference results.

(f) neutron density results generated by the LD method, with $s=0.1$ second, (scheme 1 cf. Table 1 ).

(g) relative deviations of the neutron density results generated by the LD method, with respect to the reference results, with $s=0.1$ second.

(h) neutron density results generated by the LD method, with $s=1$ second, (scheme 1 cf. Table 1).

(i) relative deviations of the neutron density results generated by the LD method, with respect to the reference results, with $s=1$ second.

\section{Concluding Remarks}

- In this paper we have described a new quasi-conservative LD finite element method for nuclear reactor point kinetics model. We remark that the extension to higher order expansions, as in [5] and [1], is straightforward.

- For the case of prescribed reactivity change as a linear function of time, we obtain the second-order moment in the first-order discretized balanced equation, and thus obtain a quasi-conservative numerical method, as we need to 
approximate it; we consider five different schemes of approximation. Model problems No. 1 illustrate that schemes 1, 3, 4 and 5 generate very accurate results for wide time step calculations.

- The quasi-conservative LD finite element method generates very accurate results in wide time step calculations as compared with recent numerical methods published in the literature, without losing significant computational efficiency.

Resumo. O modelo da cinética pontual do reator nuclear é um problema de valor inicial caracterizado por um sistema de equações diferenciais ordinárias que descreve a dependência da população do nêutrons no núcleo de um reator nuclear. Neste trabalho nós descrevemos um método de elemento finito descontínuo linear quase-conservativo para resolver numericamente as equações da cinética pontual com seis grupos de precursores dos nêutrons atrasados. O método baseia-se na expansão da solução em polinômios do Legendre em cada passo de tempo. Como nós truncamos a expansão em polinômios de Legendre do primeiro grau, nós o denominamos método descontínuo linear (LD). Nesta dedução das equações discretizadas LD nós obtivemos equações de balanço discretizadas para momentos de ordem $\ell, \ell=0,1$. Para o caso de trocas de reatividade como uma função linear do tempo, na equação do momento de primeira ordem, nós precisamos aproximar o momento de segunda ordem, desta forma nós obtivemos um esquema numérico quase-conservativo. Além disto, consideramos a expansão linear da solução em cada passo de tempo e obtivemos as equações auxiliares, que junto com as equações de balanço formam um sistema de quatorze equações lineares com quatorze incógnitas para cada passo de tempo. Mostramos os resultados numéricos de dois problemas modelos típicos para ilustrar a precisão do método LD.

\section{References}

[1] R.L. Burden and J.D. Faires, "Numerical Analysis", BROOKS/COLE Publishing Company, USA, Sixth edition, 1997.

[2] P. Engrand and P. Turrinsky, Comparison of several numerical methods for solving point kinetics equations, Transactions American Nuclear Society, $\mathbf{8 3}$ (1992), 204-206.

[3] A. Nóbrega, "Aplicação de Aproximações Racionais de Padé e Extrapolação de Richardson a Cinética de Reatores Nucleares", Rio de Janeiro, COPPE/UFRJ, 1983.

[4] P.L. Nóbrega, "Desenvolvimento de um Algoritmo Via Métodos Variacionais para Solução das Equações da Cinética Pontual", Rio de Janeiro, COPPE/UFRJ, 1995.

[5] W.M. Stacey, "Nuclear Reactor Physics", John Wiley \& Sons Inc., USA, 2001. 